Gefässchirurgie 2014 · 19:471-480

DOI 10.1007/s00772-014-1383-1

Online publiziert: 14. August 2014

(c) The Author(s) 2014. This article is published

with open access at Springerlink.com

W. Schäberle · L. Leyerer

Klinik für Viszeral-, Gefäß-, Thorax- und Kinderchirurgie, Klinik am Eichert,

Akademisches Lehrkrankenhaus der Universität Ulm, Göppingen

\title{
Strukturierte, zeiteffiziente und therapieorientierte Ultraschalldiagnostik bei Dialyseshuntproblemen
}

\begin{abstract}
störung, durch Teilthrombosierung zentraler Venen, eine zentrale Shuntstenose oder durch ausgeprägt ausgebildete von der Shuntvene abgehende Seitenastvenen, die retrograd Blut nach peripher stauen.
\end{abstract}

Shuntaneurysmen sind sonographisch eine Blickdiagnose und werden deshalb hier nicht weiter diskutiert.

\section{Untersuchungsablauf} tom, Verschluss eines Kunststoffshunts, die meist vor der Therapie keiner weiteren Diagnostik bedürfen, bestehen im Wesentlichen drei Shuntprobleme bzw. shuntassoziierte Probleme, bei denen vor adäquater Therapie eine bildgebende Diagnostik notwendig ist.

- Zum ersten ist dies der verminder-

te und für die Dialyse nicht mehr ausreichende Shuntdurchfluss mit der Fragestellung, ob ursächlich eine Shuntstenose oder eine partielle Shuntthrombosierung oder ob ein Einstromproblem mit vorausgeschalteter arterieller Stenose (A. subclavia) oder ein Abstromproblem mit zentraler Venenthrombose (V. axillaris) vorliegt.

- Das zweite shuntassoziierte Problem ist eine periphere Ischämie, meist stealbedingt durch zu hohes Shuntvolumen oder bedingt durch eine arterielle Stenose (oft A. subclavia).

- Ein weiteres shuntassoziiertes Problem ist die Armschwellung, bedingt entweder durch eine venöse Abfluss-
Die farbkodierte Duplexsonographie (FKDS) hat zwei Methoden zur Verfügung, um die Shuntprobleme ausgehend von der klinischen Fragestellung zeiteffiProblemanalyse aus Spektralanalyse zient zu erklären. Über das Grauwertbild lassen sich neben dem Shuntverlauf auch morphologische Veränderungen wie Aneurysmen oder narbige Veränderungen darstellen, weiterhin auch von der Shuntvene abgehende Seitenastvenen, die als Nebenstromleiter einen Durchfluss in der Shuntvene mindern.

Primär kommt jedoch die Beurteilung der Hämodynamik zum Einsatz, wobei duplexsonographisch der Shuntdurchfluss, aber auch der arterielle Einstrom und der Stenosegrad evaluiert werden. Eine zeiteffiziente und therapieorientierte Untersuchung wird deshalb im Einzelnen folgendermaßen nach einer eigens entwickelten 3-Punkt-Methode aufgebaut:

Tab. 1 Duplexsonographische, strukturierte Shuntfunktionsanalyse: Untersuchungsort mit

\begin{tabular}{|lll}
$\begin{array}{l}\text { Messort mit Ableitung des } \\
\text { Dopplefrequenzspektrums }\end{array}$ & Diagnostische Relevanz & $\begin{array}{l}\text { (Indirekte) Kriterien in der } \\
\text { Spektralanalyse }\end{array}$ \\
\hline $\begin{array}{l}\text { Arterie proximal der Shunt- } \\
\text { anastomose mit und ohne } \\
\text { manuelle Shuntkompression }\end{array}$ & $\begin{array}{l}\text { (Zentrale) arterielle Stenose, } \\
\text { proximal der Shunt- } \\
\text { anastomose }\end{array}$ & $\begin{array}{l}\text { Akzelerationszeit verlängert, gerin- } \\
\text { gere Pulsatilität (indirekte Kriterien } \\
\text { unter Shuntkompression) }\end{array}$ \\
\cline { 2 - 3 } & Shuntstenose & $\begin{array}{l}\text { Strömungschrakter zunehmend } \\
\text { pulsatiler (Stenosegradabhängig); } \\
\text { peripher erhöhter Widerstand }\end{array}$ \\
\hline $\begin{array}{l}\text { Arterie distal der Shuntanasto- } \\
\text { mose mit und ohne manuelle } \\
\text { Shuntkompression }\end{array}$ & $\begin{array}{l}\text { Steal-Syndrom (symptoma- } \\
\text { tisch/asymptomatisch) }\end{array}$ & $\begin{array}{l}\text { Geringe PSV < Pendelströmung } \\
<\text { retrograde Strömung entspre- } \\
\text { chend zunehmendem Steal }\end{array}$ \\
\cline { 2 - 3 } & $\begin{array}{l}\text { Periphere Durchblutungs- } \\
\text { reserve }\end{array}$ & $\begin{array}{l}\text { PSV-Zunahme (quantitativ) bei } \\
\text { Shuntkompression }\end{array}$ \\
\hline $\begin{array}{l}\text { Shunt 2-4 cm distal der } \\
\text { Anastomose }\end{array}$ & Shuntanastomosenstenose & $\begin{array}{l}\text { Intrastenotisch PSV-Zunahme } \\
\text { (Stenosegraduierung) }\end{array}$ \\
\cline { 2 - 3 } & $\begin{array}{l}\text { Zentrale Shuntsstenose/ } \\
\text { partielle Thrombose }\end{array}$ & $\begin{array}{l}\text { Strömungscharakter zunehmend } \\
\text { pulsatiler (abhängig vom Steno- } \\
\text { segrad) }\end{array}$ \\
& & \\
\hline PSVStrömungsgeschwindigkeit („peak systolic velocity). &
\end{tabular}



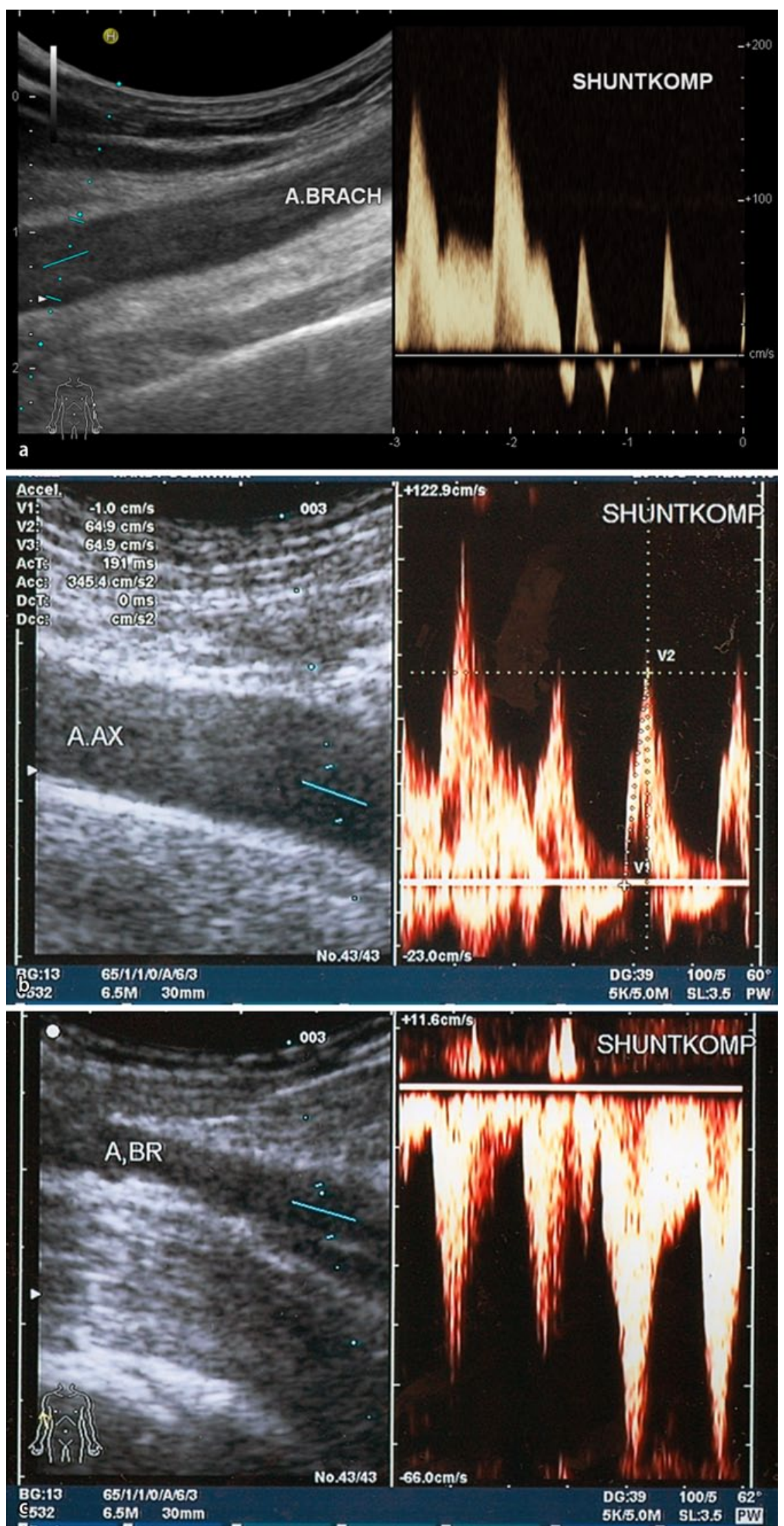

Abb. $1<$ a A brachialis mit für shuntspeisende Arterien typischem, monophasischem Dopplerfrequenzspektrum (linke Seite des Spektrums). Nach manueller Shuntkompression (rechte Seite des Spektrums: SHUNTKOMP) wird das Spektrum triphasig (Extremitätenarterie) und die indirekten Zeichen zum Ausschluss einer vorgeschalteten (proximalen) arteriellen Stenose sind verwertbar. (Bei Shuntverschluss oder hochgradiger Shuntabflussbehinderung gleiches Spektrum wie bei manueller Shuntkompression). b Patient mit ischämischen Fingerkuppennekrosen (Kaufmann Fistel): Strömungsabnahme im Dopplerfrequenzspektrum aus A. axillaris bei manueller Shuntkompression und hochgradiger A. subclavia Stenose: links im Spektrum ohne manuelle Shuntkompression; rechts zeigen bei manueller Shuntkompression (KOMP) die indirekten Zeichen eine vorgeschaltete Stenose: verzögerter systolischer Anstieg (Akzelerationszeit), reduzierte Strömungsgeschwindigkeit („,peak systolic velocity“; PSV). c Dopplerfrequenzspektrum aus A. brachialis distal der Shuntanastomose: monophasisch poststenotisch. Flusszunahme bei manueller Shuntkompression (rechter Teil des Dopplerspektrums, KOMP) bei weiterhin poststenotischem Strömungssignal 
Zunächst wird die A. brachialis am Oberarm im Querschnitt aufgesucht und im Längsschnitt das Dopplerfrequenzspektrum abgeleitet. Dieses zeigt normalerweise den shuntbedingten hohen diastolischen Anteil des monophasischen Strömungssignals. Wenn dieses pulsatiler erscheint oder gar das für Extremitäten typische triphasische Strömungssignal aufweist, besteht ein Shuntdurchflussproblem (Shuntverschluss oder hochgradige Shuntstenose) oder ein Abstromproblem der zentralen Venen (V.-axillaris-Thrombose).

Im nächsten Schritt wird das Dopplerfrequenzspektrum in der A. brachialis bei manueller Shuntkompression beurteilt (- Tab. 1, - Abb. 1, Video1). Wenn es nicht den triphasischen Strömungscharakter mit steilem systemischem Anstieg (kurze Akzellerationszeit) und den peitschenden Klang zeigt, ist von einer vorgeschalteten Stenose der shuntspeisenden Arterie auszugehen (meist A.-subclaviaStenose). Diese ist durch kontinuierliche Untersuchung der proximaleren arteriellen Strombahn darzustellen und zu graduieren.

Der zweite Messpunkt mit Ableitung und Beurteilung des Dopplerfrequenzspektrums ist die Leitarterie distal der Shuntanastomose mit und ohne Shuntkompression (eventuell bei Ischämie auch Fingerarterien) zur Beurteilung eines Steal-Syndoms durch die AV-Fistel.

Der dritte Messpunkt liegt im Shunt selbst $2 \mathrm{~cm}$ distal der Shuntanastomose zur Beurteilung des Shuntflusses und von Anastomosenstenosen. Die weiteren diagnostischen Schritte in der Ultraschalluntersuchung richten sich nach der klinischen Fragestellung bzw. dem Shuntproblem in Zusammenhang mit der Spektralanalyse an den beschriebenen Punkten.

\section{Problem: unzureichender Shuntdurchfluss}

Bei reduziertem, für die Dialyse unzureichendem Shuntfluss und pathologischem, zu pulsatilem Strömungssignal in der A. brachialis muss das Strömungshindernis im Shunt gesucht werden und es wird dabei, insbesondere bei Ciminofisteln, zunächst die Anastomose dargestellt. Wenn diese unauffällig ist, muss im Ver-

Gefässchirurgie 2014 · 19:471-480 DOI 10.1007/s00772-014-1383-1

(c) () The Author(s) 2014. This article is published with open access at Springerlink.com

\section{W. Schäberle · L. Leyerer \\ Strukturierte, zeiteffiziente und therapieorientierte Ultraschalldiagnostik bei Dialyseshuntproblemen}

\section{Zusammenfassung}

Hintergrund und Fragestellung. Eine gute Shuntfunktion ohne Beeinträchtigung des Patienten ist oberstes Ziel in der Shuntchirurgie. Die wesentlichen Shuntprobleme (zu geringer Shuntfluss, periphere Ischämie, Armschwellung, Shuntinfekt) erfordern ein differenziertes diagnostisches Vorgehen zur Klärung des oft komplexen hämodynamischen Problems. Die rein morphologische Betrachtung wird dem Shuntproblem oft nicht gerecht.

Material und Methoden. Anhand der aktuellen Literatur und eigener Erfahrungen wird eine strukturierte, zeiteffiziente und therapieorientierte Ultraschalldiagnostik dargestellt.

Ergebnisse und Schlussfolgerungen. Die Duplexsonographie ist eine valide Methode in der Diagnostik von shuntassoziierten
Problemen und Shuntstenosen. Ein strukturiertes Vorgehen mit Analyse des punktuell abgeleiteten Dopplerfrequenzspektrums aus der shuntspeisenden Arterie vor der Shuntanastomose und der Arterie knapp distal der Anatomose ohne und mit manueller Shuntkompression sowie der arteriovenösen Fistel kurz nach Anastomose ermöglicht unter Würdigung des klinischen Shuntproblems das gezielte sonographische Aufsuchen der morphologischen oder hämodynamischen $\mathrm{Pa}$ thologie. Die Untersuchung kann mit einer sehr hohen Treffsicherheit in weniger als 10 min durchgeführt werden.

Schlüsselwörter

Duplexsonographie · Dialyseshunt · StealSyndrom · Shuntfluss · Shuntkomplikationen

\section{Structured, time-efficient and therapy-oriented ultrasonography diagnostics for dialysis shunt problems}

\section{Abstract}

Background and aims. A good shunt function without negative effects on the patient is the main priority of shunt surgery. The main shunt problems, e.g. shunt flow too low, peripheral ischemia, arm swelling and shunt infection, necessitate a differential diagnostic approach to clarify the often complex hemodynamic problem as a purely morphological view is often insufficient to deal with the shunt problem.

Material and Methods. A structured, timeefficient and therapy-oriented ultrasonography diagnostics is described based on the current literature and own experience.

Results and conclusions. Duplex Doppler ultrasonography is a valid method in the diagnostics of shunt-associated problems and shunt stenoses. A structured approach with analysis of the selectively derived Doppler frequency spectrum from the artery feeding the shunt in front of the shunt anastomosis and the artery immediately distal to the anastomosis, with and without manual shunt compression as well as the arteriovenous fistula shortly after the anastomosis allows a targeted sonographic search for the morphological or hemodynamic pathology under appraisal of the clinical shunt problem. The examination can be performed with a very high accuracy in less than 10 minutes.

\section{Keywords}

Doppler duplex ultrasonography · Dialysis shunt $\cdot$ Steal syndrome Shunt flow . Complications lauf nach Shuntstenosen oder einer partiellen Shuntthrombose gesucht werden. Wenn im Shuntverlauf weiterhin ein $\mathrm{zu}$ pulsatiles Strömungssignal vorherrscht, so ist eine zentrale Abflussstörung anzunehmen und insbesondere die V. axillaris und V. subclavia auf eine Thrombose oder partielle Thrombose (• Abb. 2) hin zu untersuchen oder nach einer Mündungsstenose der V. cephalica in die V. axillaris zu suchen.
Shuntstenosen (Video 2) sind meist an der Shuntanastomose (in $>60 \%$ ) lokalisiert [10] bei älteren AV-Fisteln auch vor und nach aneurysmatischen Veränderungen oder aufgrund narbiger Stenosen im Punktionsbereich. Hier wird der grauwertsonographisch gemessene stenotische Restdurchmesser von $<2 \mathrm{~mm}$ als Kriterium für eine drohende Shuntthrombose dargestellt, die hämodynamische Stenosebeurteilung ist jedoch zuverlässiger. 


\section{Vaskuläre Sonographie}
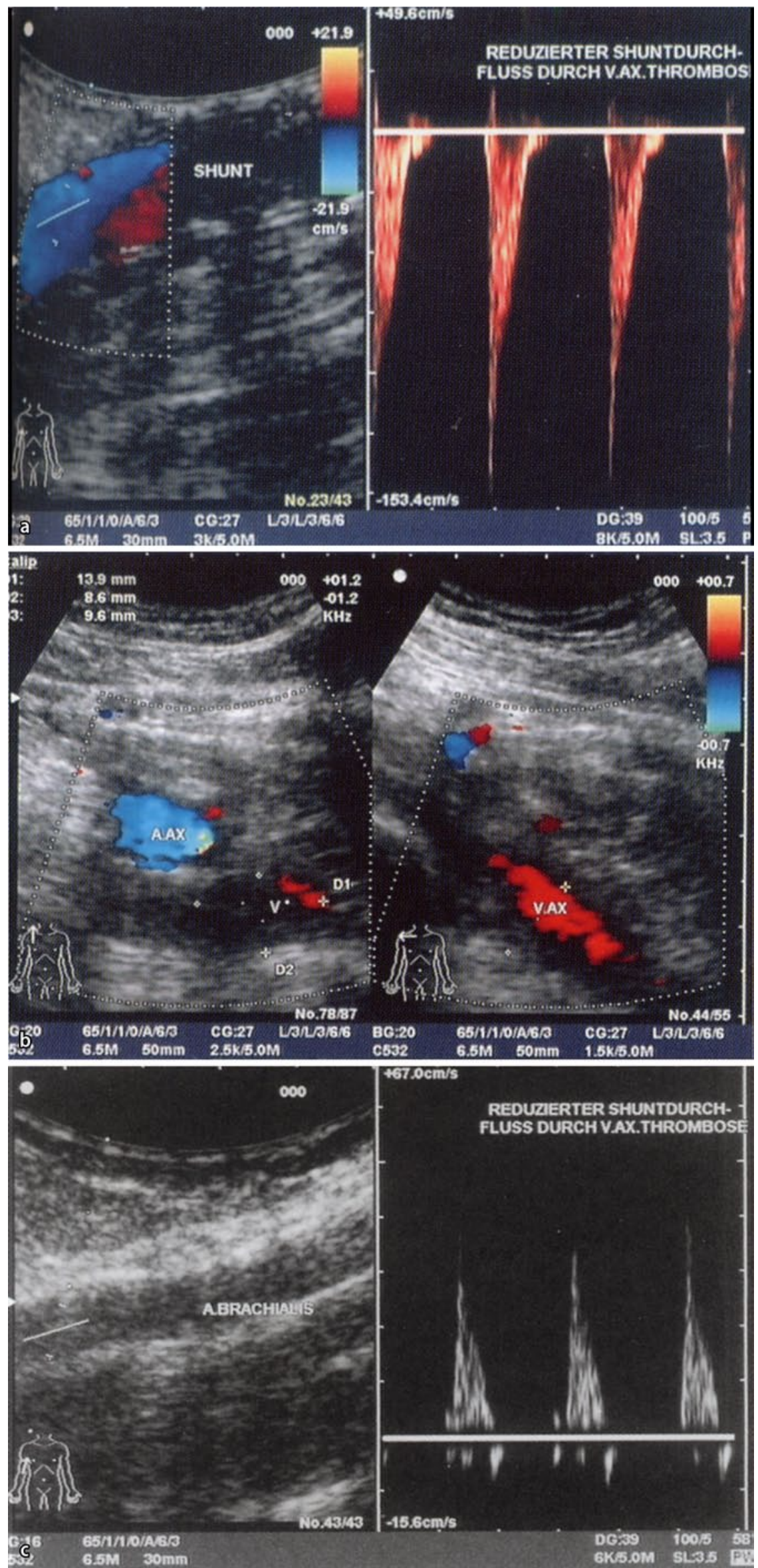

Abb. $2<$ Unzureichendes Shuntvolumen. a Kunststoffinterponat mit pulsatilem Dopplerfrequenzspektrum ohne shunttypischen diastolischen Strömungsanteil. b Reduziertes Shuntflussvolumen durch umflossene Axillarvenenthrombose (V. axillaris links im Querschnitt, rechts im Längsschnitt: weitgehend thrombosiert, randständiges Strömungssignal rot kodiert). c Shuntspeisende Arterie mit typischem Spektrum (pulsatil) für Abflussbehinderung oder Stenose (z. B. V.-cephalica-Mündung) im venösen herzwärts gerichteten Shuntareal. Zentrale Thrombose führt im Gegensatz zu diesem Patienten klinisch oft zum Problem Armschwellung. (Aus [12]) 

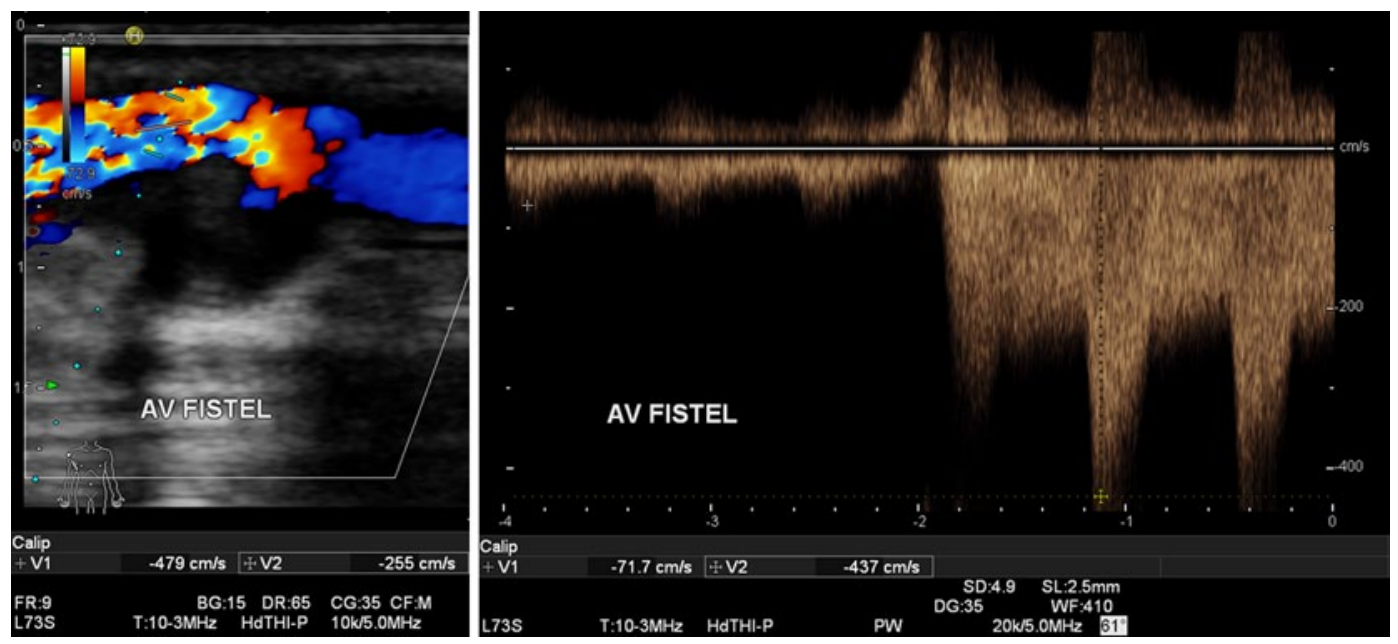

Abb. $3 \Delta$ Hochgradige Shuntstenose PSV-Ratio $=6$ (links intrastenotisch $437 \mathrm{~cm} / \mathrm{s} /$ rechts prästenotisch $71 \mathrm{~cm} / \mathrm{s}$ ). Spektrum erzeugt durch kontinuierliches Verschieben des Transducers auf der Haut von prä- zu intrastenotisch. Stenose (Intimaflap und Shuntkompression) verursacht durch weitgehend thrombosiertes, punktionsbedingtes Aneurysma spurium (blau kodiert). Therapeutische Konsequenz aus Ultraschallbefund: nicht perkutane transluminale Angioplastie (PTA), sondern chirurgische Revision

Die Stenosegraduierung, anhand von aus der Diagnostik peripherer arterieller Stenosen bekannten Grenzgeschwindigkeiten, ist bei Shuntstenosen meist ungenau, weil neben den zentralen Einflussgrößen wie Blutdruck der vom Shuntlumen abhängige Durchfluss eine schwer zu wertende Einflussgröße ist. Ein verlässlicherer Parameter ist daher die sprunghafte Erhöhung der prästenotischen zur intrastenotischen Strömungsgeschwindigkeit (Quotient intrastenotisch/prästenotisch). In der arteriellen Strombahn ist auch hier bei der sprunghaften Erhöhung auf das doppelte von einer $50 \%$ Stenose auszugehen und bei einer Erhöhung auf das 4-Fache von einer höhergradigen, $>75 \%$ igen Stenose (konzentrische Stenose angenommen). Im Shuntverlauf besteht bei einem Quotienten von über 3 eine hämodynamisch relevante Stenose (• Abb. 3, Video 3). Die hämodynamische Relevanz von Shuntstenosen sagt jedoch noch nichts über die therapeutische Relevanz von Stenosen aus. Für den dialyserelavanten Blutfluss werden erst höhergradige Quotienten (PSV-Ratio von 4 bis 8) therapierelevant und hier sind ergänzende Parameter wie ein zunehmend pulsatiler Fluss in der shuntspeisenden Arterie (- Abb. 2, 3, 4, Video 4) und eine Abnahme des Blutflussvolumens (gemessen in der A. brachialis) bedeutsam [12]. Der Quotient ist jedoch auch nur bei relativ konstanten Shuntdurchmessern, ohne prästenotisch ausgeprägte Ektasien verwertbar (alternativ Stenosedurchmesserbestimmung).

Bei unauffälligem, monophasischem Strömungssignal in der A. brachialis sowie im Shunt, wird der Shuntverlauf im Querschnitt gemappt und nach von der Shuntvene abgehenden Seitenästen, insbesondere nach großlumigeren, gesucht, die als ineffiziente Parallelleiter fungieren. Diese Seitenäste können dann entsprechend der sonographischen Markierung (im Hautniveau) zur Shuntflussverbesserung gezielt operativ aufgesucht und ligiert werden.

\section{Problem: periphere Ischämie}

Die Ursachenforschung bei peripherer Ischämie (in 2-8\% der Dialysschunts) kann sich komplexer gestalten. Eine zentral der Shuntanastomose gelegene relevante, arterielle Stenose, die auch mit dem Problem einer unzureichenden Shuntdurchblutung einhergehen kann, zeigt bei Ausschalten des Shunteinflusses unter manueller Shuntkompression im proximal der Anastomose abgeleiteten Dopplerfrequenzspektrum die indirekten Stenosezeichen (verzögerte Akzelerationszeit, monophasisches Strömungssignal - Abb. 1). Die Stenoselokalisation geschieht dann durch Mappen des vorgeschalteten Arterienabschnitts.
Für die adäquate Therapie beim StealSyndrom ist die Shunthämodynamik entscheidend. Dazu wird als nächster Schritt das Dopplerfrequenzspektrum aus der Arterie knapp distal der Shuntanastomose abgeleitet. Der arterielle Fluss mit und ohne manuelle Shuntkompression wird verglichen. Wenn das Strömungssignal distal der Shuntanastomose ohne Shuntkompression eine Pendelströmung (systolisch orthograder Strömungsrichtung, diastolisch retrograder Strömungsrichtung) oder gar ein komplett retrogrades Strömungssignal zeigt, so ist von einem Steal-Phänom auszugehen (• Abb. 4, Video 4). Dabei wird die shuntspeisende Arterie über andere Leitarterien (A. ulnaris, A. interossea, A. brachii profunda) zusätzlich retrograd perfundiert. Dies kann symptomfrei oder symptomatisch verlaufen. Bei symptomatischem Steal-Syndrom mit peripherer Ischämie muss zwischen hyper-, normo- (bei autologem Shunt $400-800 \mathrm{ml} / \mathrm{min}$ ) und hypodynamem Shunt differenziert werden. Bei therapierelevanter Ischämie kann die adäquate Therapie über die sonographische Shuntflussbestimmung [12] festgelegt werden: bei hyperdynamem Shunt shuntflussreduzierende Verfahren („shunt banding“, lumenreduzierendes Interponat, RUDI) und bei normo-/hypodynamem Shunt periphere Durchblutungsverbesserung durch DRIL, DRAL oder PAVA [1, 5, 11, $13,17]$. 

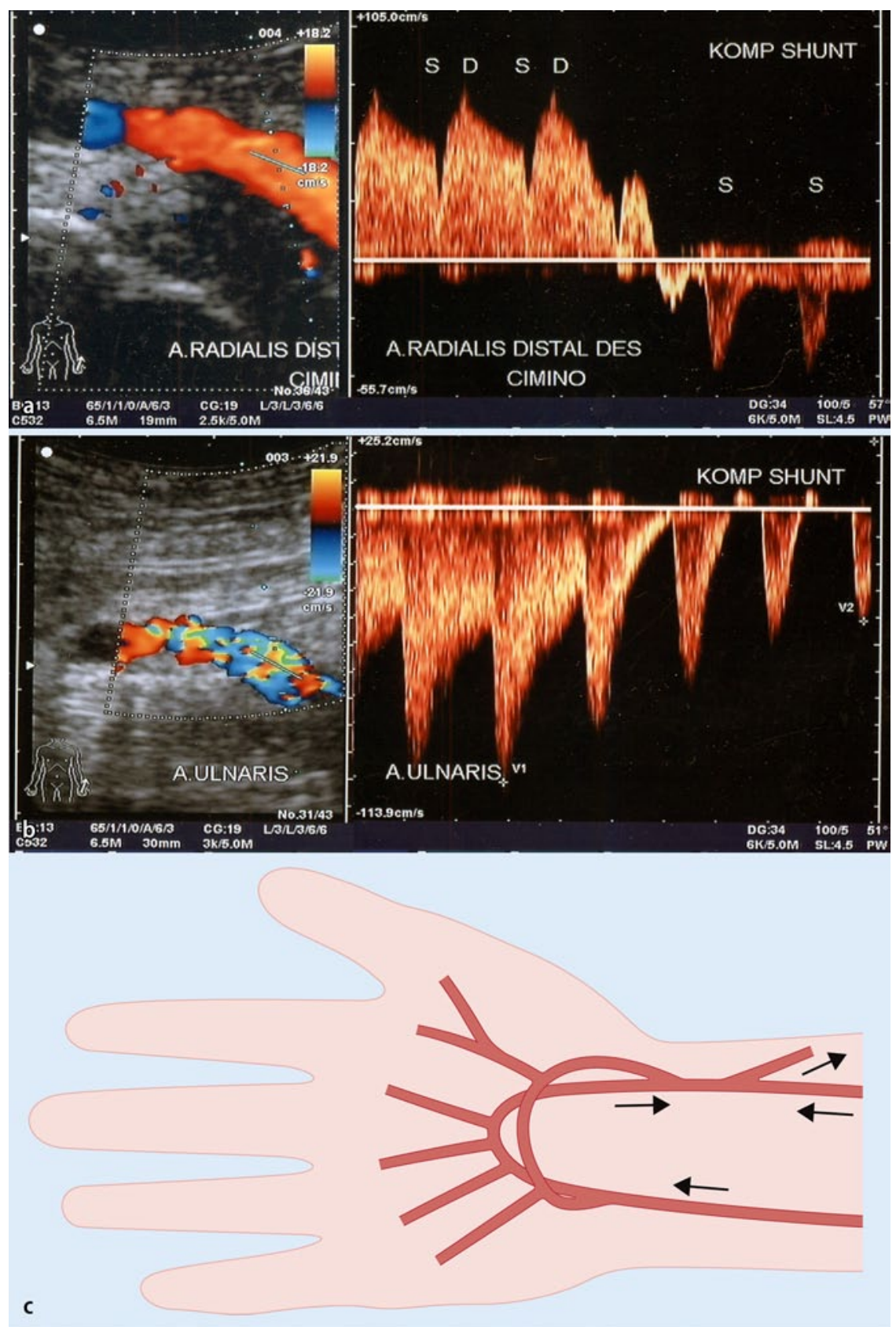

Abb. $4 \Delta$ a Periphere Ischämie (Diabetiker) bei normodynamem Shunt mit Steal-Syndrom. Retrograder Fluss in die A. radialis distal des Ciminoshunts am Handgelenk (Shuntanastomose an blau kodierter Stelle): Im Dopplerfrequenzspektrum (linker Abschnitt) retrogrades Strömungssignal (vom Hohlhandbogen zur Shuntanastomose) mit systolischer Entschleunigung (S) und retrogradem diastolischem Fluss, jedoch bei manueller Shuntkompression (rechter Abschnitt: KOMP-Shunt) orthogrades Strömungssignal mit diastolischem Anteil bei maximaler Weitstellung der Arteriolen bei peripherer Ischämie. b Dopplerfrequenzspektrum in der A. ulnaris ohne Shuntkompression (linker Teil des Spektrums) mit shuntversorgendem Fluss-Signal (hohe Strömungsgeschwindigkeit [PSV], monophasisch) und bei manueller Shuntkompression (rechter Abschnitt, KOMP) mit pulsatilem deutlich reduziertem Strömungssignal. (vgl. Video 4). Bei dem älteren, multimorbiden Patienten konnte aufgrund des sonographischen Befunds die A. radialis distal der Shuntanastomose am Handgelenk ligiert werden ("distal radial artery ligation“; DRAL). Keine weiteren Ischämiesymptome, keine Beeinträchtigung der Shuntfunktion. c Schemazeichnung zur Illustration der Flussverhältnisse bei Steal-Syndrom; Strömungsrichtung durch Pfeile dargestellt, Pfeilmarkierung entspricht auch Messort nach der zeiteffizienten 3-Punkte-Methode (• Tab. 1)
Zwischen hyperdynamen oder normo-/hypodynamen Shunts kann durch duplexsonographische Blutflussmessungen differenziert werden. Dafür wird die grauwertsonographisch bestimme Querschnittsfläche mit der duplexsonographisch bei spitzwinkligem Dopplerwinkel $\left(<50^{\circ}\right)$ bestimmten und vom Ultraschallgerät errechneten, gewichteten mittleren Strömungsgeschwindigkeit multipliziert. Flussmessungen im Shunt sind bedingt durch die oft starken Lumenschwankungen von Ciminofisteln unzuverlässig. Weiterhin sind sie oft quer ovalär und Fehler in der Durchmesserbestimmung gehen in die Messung des Blutflusses im Quadrat ein. Shuntflussmessungen, die durch den Vergleich des Blutflusses in der Arterie proximal der Shuntanastomose und distal der Shuntanastomose verglichen werden, sind auch deshalb ungenauer, weil schon kleine Messfehler insbesondere im Durchmesser der distalen dünnlumigen Arterie (wie die distale A. radialis) zu großen Messfehlern führen. Deshalb liefert der Seitenvergleich der gut darstellbaren und gut vermessbaren A. brachialis in Oberarmmitte die validesten Ergebnisse [12]. Nach beidseitiger Bestimmung der mittleren Strömungsgeschwindigkeit wird die Querschnittsfläche sehr exakt dadurch bestimmt, dass die systolische/diastolische Lumenschwankung durch Verrechnung von 1/3 des Durchmessers in der Systole und 2/3 des Durchmessers in der Diastole (abhängig von der Zeitdauer systolisch, diastolische Durchmesser) gewichtet wird und der Durchmesser selbst nach der Leading-edge-Methode bestimmt wird, um den Messfehler durch Blooming-Effekt an Grenzflächen hoher akustischer Impedanz (Gefäßwand) im Grauwertbild zu minimieren [12]. Gleichartig wird auch der Shuntdurchfluss bei zu geringem Shuntvolumen, z. B. durch noch fehlende Shuntreife, bestimmt. Shuntflüsse mit weniger als $300 \mathrm{ml} / \mathrm{min}$ reichen für eine effektive Dialyse nicht mehr aus, und geringe sowie im Verlauf sinkende Shuntvolumina zeigen auch ein hohes Risiko eines Shuntverschlusses (bei Kunststoffloops $<700 \mathrm{ml}$ ([7, 17]). Shuntflüsse [2] über $1200 \mathrm{ml} /$ min gefährden eine periphere Ischämie sowie eine Herzinsuffizienz. Meist ist jedoch die therapeutische Option des Ste- 

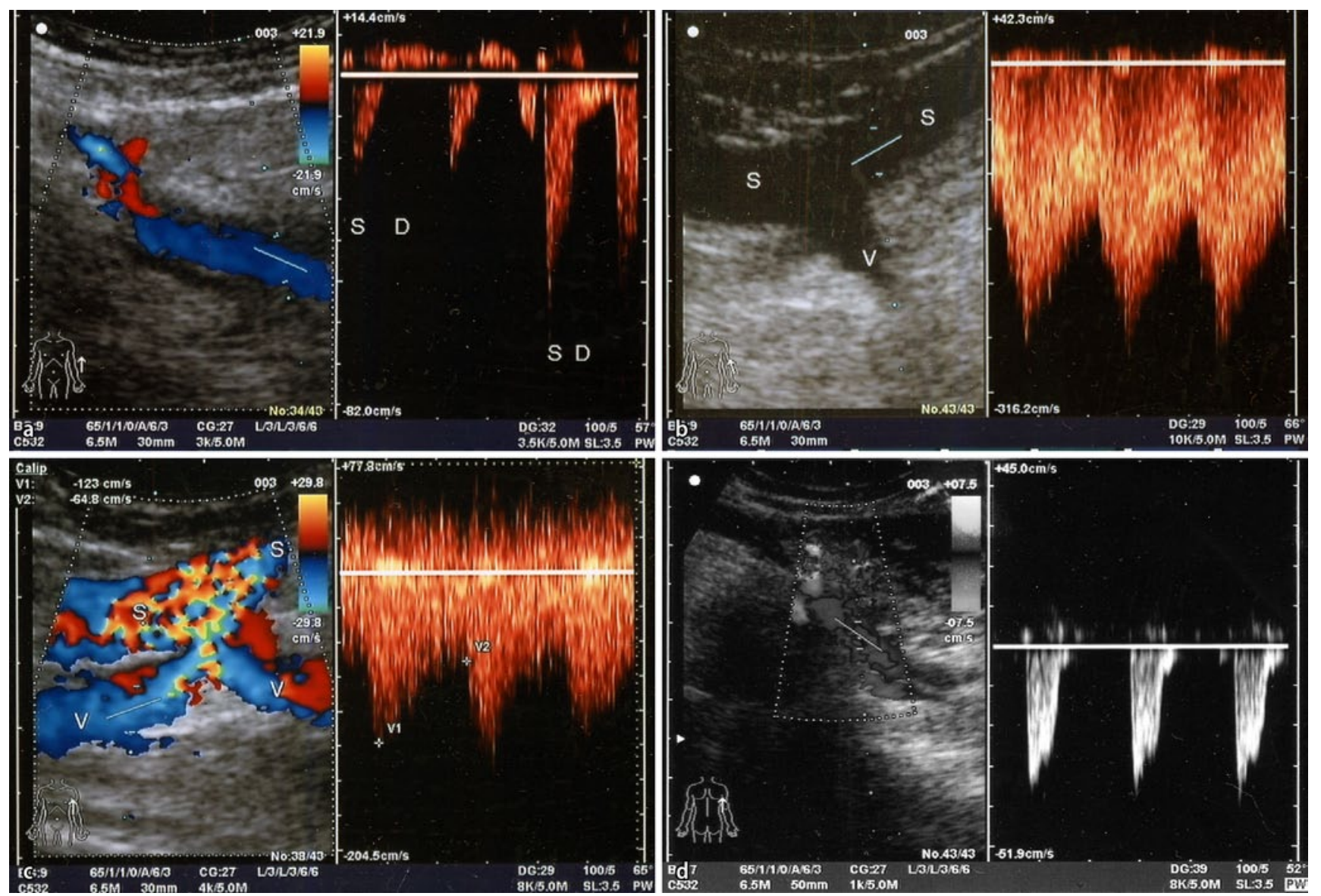

Abb. $5 \Delta$ a Patient mit peripherer Ischämie: Dopplerfrequenzspektrum aus distaler A. brachialis; distal der Shuntanastomose in Ellenbeuge (Kaufmann Fistel): Wechsel von Pendelströmung (links) in orthograde Strömung (S: Systole, D: Diastole) nach manueller Shuntkompression (rechts) mit monophasischem Spektrum (wegen peripherer Ischämie nicht triphasisch); PSVVerdopplung. b Dopplerfrequenzspektrum aus Shunt (V. cephalica) mit typischem Shuntsignal (monophasisch) und mit hoher Strömungsgeschwindigkeit (PSV) $=2,5 \mathrm{~m} / \mathrm{s}$ vor Abgang der großlumigen venöse Seitenäste (V). $\mathbf{c}$ Farbduplexonographische Darstellung (wie 4a) mit Dopplerfrequenzspektrum aus großlumig abgehendem Venenast (mit PSV $=120 \mathrm{~cm} / \mathrm{s}$ hohe Strömungsgeschwindigkeit). d Die periphere Ischämie war nach selektivem Ligieren der großlumigen venösen Seitenäste (nach sonographischer Markierung) beseitigt. Danach zeigt das Dopplerfrequenzspektrum in der A. brachialis, distal der Shuntanastomose in der Ellenbeuge eine orthograde Strömung (Verdopplung der PSV). Derartige Seitenäste können auch zum Problem „Armschwellung“ und zum Problem „nicht genügend Blutfluss in Shuntpunktionsvene“ durch Fluss in Parallelleiter führen

al-Syndroms anhand der Spektralanalyse in der Arterie distal der Shuntanastomose (sowie Fingerarterien) mit und ohne manuelle Shuntkompression beurteilbar (• Abb. 4).

Bei orthograden Strömungsverhältnissen ohne stealtypischem Fluss in der Arterie distal der Anastomose ist fast immer bei manueller Shuntkompression eine Zunahme der Strömungsgeschwindigkeit („peak systolic velocity“; PSV) und somit der Durchblutung zu detektieren. Davon abhängig ist zu werten, ob eine dosierte Durchblutungsminderung im Shunt zur ausreichenden Mehrdurchblutung der Hand führt und ob eine Shuntdrosselung („shunt banding“ oder lumenreduzieren- des Interponat, meist nur bei im Shunt gemessener hoher PSV $>2 \mathrm{~m} / \mathrm{s}$ ) sinnvoll ist. Zur Kontrolle des Therapieeffekts kann prä- wie auch intraoperativ der Shunt partiell komprimiert werden und darunter der Fluss in der distalen Arterie und im Shunt beurteilt werden. Wichtig ist jedoch zuvor die Klärung, ob distal der Anastomose (insbesondere bei Diabetikern) im weiteren Verlauf bis zu den Fingerarterien arterielle Stenosen vorliegen. Hilfreich ist dafür eine etappenweise Darstellung des Dopplerfrequenzspektrums in der A. radialis distal sowie der Fingerarterien mit intermittierendem Mappen. Diese Stenosesuche ist aufwendiger und insbesondere beim Diabetiker mit ausgeprägter Me- diaverkalkung eingeschränkt, sodass bei dieser Fragestellung eine ergänzende angiographische Untersuchung hilfreich ist. Bei allen anderen Fragestellungen ist jedoch eine angiograpische Untersuchung nicht notwendig. Die gezielte farbduplexsonographische Untersuchung beurteilt über die Hämodynamik das Shuntproblem meist besser.

Ein ineffizient hohes Shuntvolumen kann selten auch durch lumenstarke, von der Shuntvene abgehende venöse Seitenäste entstehen, die derartig viel Blutvolumen abziehen, dass die periphere Ischämie nach Ligatur dieser Seitenäste (präoperativ sonographische Markierung) beseitigt ist (• Abb. 5). 

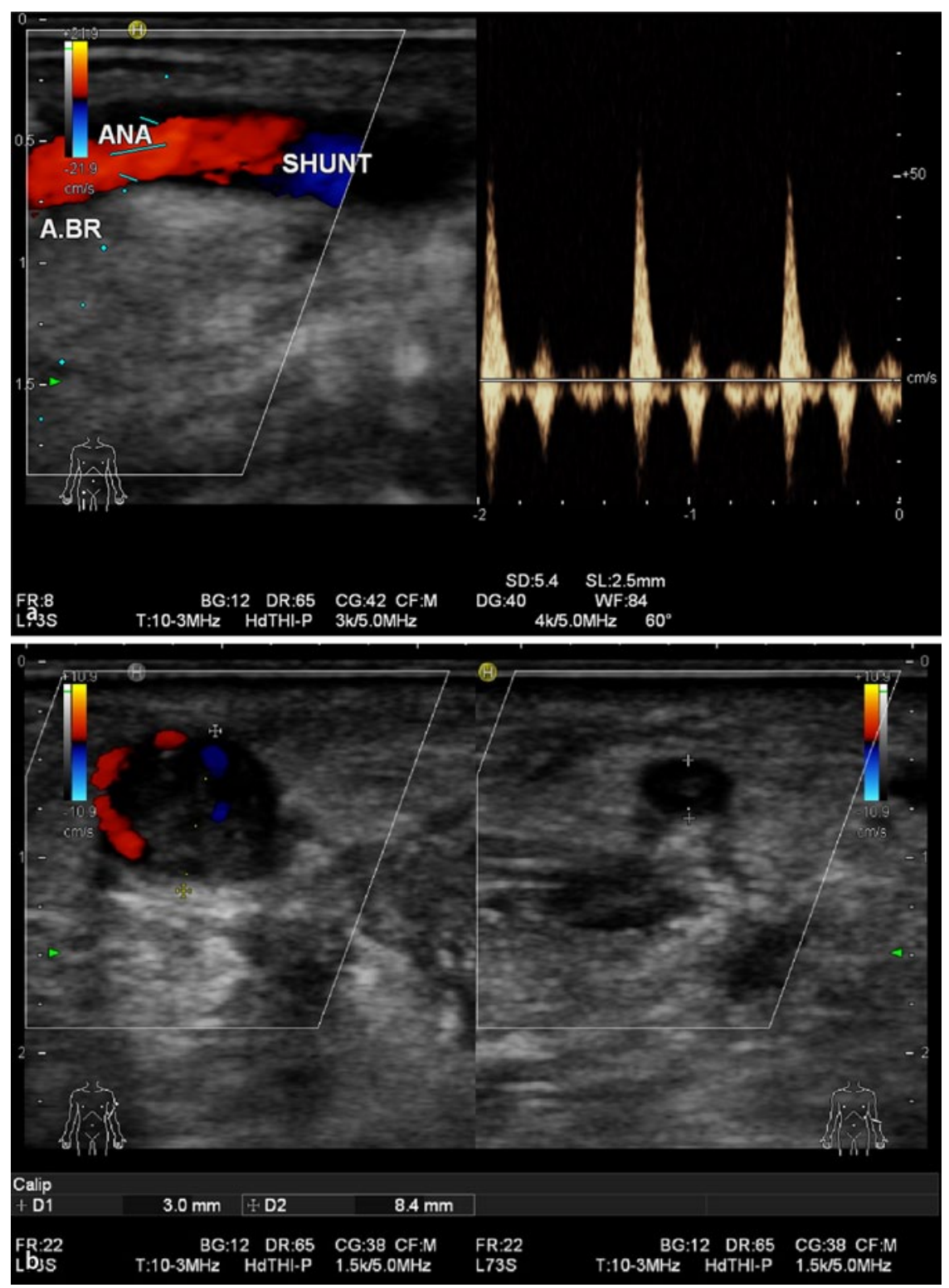

Abb. $6 \Delta$ a Dialyse trotz pulsierendem Shunt nicht mehr möglich. Dopplerfrequenzspektrum aus AV Fistel in der Ellenbeuge knapp distal der Anastomose, sehr pulsatil, triphasisch. Spektrum aus zuvor dargestellter A. brachialis (nicht dargestellt) triphasisch (wie in - Abb. 2b). b Links im Querschnitt partielle Thrombosierung der AV-Fistel ca. $10 \mathrm{~cm}$ von der Anastomose entfernt mit Randflussphänomen, Randfluss darstellbar nur bis Abgang eines venösen Seitenastes darstellbar, dann Verschluss der Shuntvene (V. cephalica). Rechts im Bild dünnlumige verschlossene Shuntvene knapp oberhalb des noch offenen abgehenden Seitenastes, Durchmesser $3 \mathrm{~mm}$ als Zeichen eines Verschlusses bei narbiger Enge bei langjähriger Punktion, somit kein radiologisch interventionelles Verfahren zur Therapie sinnvoll

\section{Problem: Armschwellung}

Die shuntassoziierte Armschwellung zeigt bei (partieller) Thrombose der zentralen Venen oder einer Cephalikamündungsstenose (Video 5), meist eine erhöhte Pulsatilität in der anastomosennahen Shuntvene. Kompressionssonographisch oder farbduplexsonographisch geschieht der Thrombosenachweis in der Mohren- die als Nebenleiter oft Blut nicht nur nach zentral transportieren, sondern verlaufsabhängig auch retrograd in Unterarm und Handvenen pumpen können und in denen dann ein Blutfluss mit Strömungsrichtung nach peripher und in Richtung Hand dargestellt werden kann. Diese werden dann nach sonographischer Markierung gezielt operativ ligiert.

Andere Komplikationen wie Punktionsaneurysmen sind farbduplexsonographisch eine Blickdiagnose. In der Shuntleckage kann die typische Pendelströmung duplexsonographisch nachgewiesen werden. Zur Aneurysmaausschaltung ist die ultraschallgesteuerte Thrombininjektion wie beim punktionsbedingten Aneurysma spurium bei Femoralarterienpunktion möglich. Sie sollte jedoch zur Sicherheit unter kurzfristiger manueller Shuntkompression zentral des Aneurysmas während der Thrombininjektion durchgeführt werden, um eine Thrombineinschwemmung nach zentral zu verhindern.

\section{Ultraschallbasierte Therapie}

Hilfreich kann die Farbduplexsonographie auch in der Therapiewahl (operativ gegen endovaskulär) bei verschlossenen Ciminofisteln sein. So können auch an adipösen Armen Shunts mit aneurysmatischen Erweiterungen und partiellen Shuntengen gut beurteilt werden. Weiterhin kann auch beim Shuntverschluss die Beurteilung des Shunts im Verlauf narbige längerstreckige Verengungen anhand dünnlumiger, zum Teil echoreicherer Shuntareale zeigen ( $\bullet$ Abb. 6, Video 6). Auch Shuntektasien/-aneurysmen mit partieller thrombotischer Wandauskleidung und durch Streuung bedingte Verschlüsse benötigen eine offen-chirurgische Revision und können sonographisch gut beurteilt werden.

Die Stenose ist bei älteren Ciminofisteln wegen starker Lumenschwankungen (Wechsel zwischen aneurysmatischen Erweiterung und oft narbigen Engen) oft schwieriger zu graduieren, insbesondere weil diese Lumenschwankung noch höhere Quotienten (von aneurysmatischer Erweiterung zu narbiger Enge) aufweist, ohne dass dadurch eine dialyserelevante Durchblutungsminderung und somit 


\section{Detaillierte Beschreibung zu den Videos}

\section{Video 1}

Das in der A. brachialis abgeleitete Dopplerfrequenzspektrum zeigt den typischen hohen diastolischen Fluss einer shuntspeisenden Arterie. Nach manueller Shuntkompression, also nach Ausschalten des AV-Fisteleinflusses, verändert es sich zum typischen triphasischen Spektrum einer Extremitätenarterie.

Video 2

Anastomosenstenose (Unterarmloop - V. basilika). Der umschriebene PSV-Anstieg von $100 \mathrm{~cm} / \mathrm{s}$ prästenotisch auf knapp $400 \mathrm{~cm} / \mathrm{s}$ drückt eine $70 \%$ Stenose aus.

Video 3

Stenose im Shuntverlauf (Punktionsbereich der Ciminofistel) verursacht durch ein Punktionsaneurysma (weitgehend thrombosiertes Aneurysma spurium). Der PSV-Anstieg von $100 \mathrm{~cm} / \mathrm{s}$ auf intrastenotisch $433 \mathrm{~cm} / \mathrm{s}$ drückt eine $75 \%$ Stenose aus (PSV-Ratio >4)

Video 4

Aus A. radialis abgeleitetes, für shuntspeisende Arterien typisches, monophasisches Dopplerfrequenzspektrum mit hohem diastolischen Anteil bei Ciminofistel am Handgelenk. Bei Transducerpositionswechsel von proximal der Shuntanastomose zu distal der Shuntanastomose (Strömungsrichtung normal vom Transducer weg nach peripher) zeigt die distale A. radialis (distal der Shuntanastomose) nicht das typische triphasige Fluss-Signal, sondern ein monophasisches retrogrades Strömungssignal (auf den Transducer zu). Die distale A. radialis ist somit über die A. ulnaris und den arteriellen Hohlhandbogen fistelversorgend, was oft zu periphärer Ischämie führt (Steal-Syndrom), jedoch auch, wie bei diesem Patienten, klinisch unauffällig verlaufen kann (normaler Shuntfluss, keine Ischämie)

Video 5

Bei Ciminofistel in der Ellenbeuge ist im aus der A. axillaris abgeleiteten Dopplerfrequnzspektrum die etwas erhöhte Pulsatilität (reduzierter diastolischer Flussanteil) auffallend. Diese erhöhte Pulsatilität ist Ausdruck eines erhöhten peripheren Widerstand bzw. Shuntwiderstand, in diesem Fall durch die hochgradige Stenose an der V.-cephalica-Mündung (Aliasing). Beim Platzieren des Dopplerfrequenzspektrums im Übergang V. cephalica zu V. axillaris zeigt die PSV von $5 \mathrm{~m} / \mathrm{s}$ eine hochgradige Stenose. (Am Clipende poststenotischer Fluss aus V. axillaris).

Video 6

Sehr pulsatiles Spektrum aus der A. brachialis signalisiert den Shuntverschluss der in der Ellenbeuge angelegten AV-Fistel. Die AV-Fistel ist jedoch am Abgang offen, zeigt aber ebenfalls das pulsatile Spektrum als Zeichen einer Abstrombehinderung im weiteren Verlauf. Bei weiterer Untersuchung im Shuntverlauf partielle Shuntthrombosierung (Strömungssignal neben umflossenem Thrombus) und nach weiterem Abgang eines venösen Seitenastes kompletter Shuntverschluss. Der Shuntdurchmesser schrumpft jedoch von $8 \mathrm{~mm}$ auf $3 \mathrm{~mm}$ als Ausdruck einer narbigen Enge im Punktionsbereich. Zur Shuntrekanalisation ist ein radiologisch interventionelles Verfahren inadäquat und eine operative Revision mit Interponat der narbigen Enge ist indiziert.

eine therapierelevante Stenose auftritt. Bei für die Dialyse zu geringem Blutfluss kann jedoch die Therapierelevanz der höhergradigen Stenosen beurteilt werden. Im Kunststoffloop ist bei gleichbleibendem Graftdurchmesser eine Stenose im Shuntverlauf anhand der sprunghaften Erhöhung der Strömungsgeschwindigkeit sehr gut graduierbar. Eine Stenose an der Anastomose der Ciminofistel oder eines Kunststoffloops kann jedoch über die Bildung eines Quotienten (intrastenotische/ prästenotische PSV) nicht adäquat graduiert werden, weil hier völlig unterschiedliche Gefäße miteinander verglichen werden. Es muss auf Absolutgeschwindigkeiten als Grenzgeschwindigkeit zurückge- griffen werden, wobei über $2,5 \mathrm{~m} / \mathrm{s}$ von einer Stenose ausgegangen wird. Über deren Therapierelevanz ist dabei jedoch noch nichts ausgesagt, weil bei für die Dialyse ausreichendem Shuntdurchfluss diese relative Stenose sogar sinnvoll sein kann, um eine periphere Ischämie durch ein Steal-Syndrom zu vermeiden. So erzeugt eine Dilatation einer relevanten Shuntstenose oft eine periphere Ischämie, wenn präinterventionell die Spektralanalyse schon eine Pendelströmung in der Arterie distal der Shuntanastomose zeigt. Dies zeigt die Wichtigkeit der engen Verknüpfung von sonographisch diagnostizierten hämodynamischen Parametern mit der Klinik des Patienten bzw. dem Dialyseshunt- problem, um adäquate Therapieentscheidungen zu treffen.

In einer Zwischenauswertung einer eigenen laufenden Untersuchung konnte auf Basis der o. g. 3-Punkte-Untersuchungsmethode bei 51 Patienten (68\% Ciminofistel, 32\% „loop“ oder „straight graft") in $92 \%$ eine adäquate Therapieentscheidung für das jeweilige Shuntproblem (37\% Ischämie, 53\% unzureichender Shuntfluss, 10\% Armschwellung) zeiteffizient (Untersuchungsdauer durchschnittlich $8 \mathrm{~min}$ ) ermittelt werden (Publikation in Vorbereitung).

\section{Ultraschallbasierte Surveillance-Programme?}

Ob eine routinemäßige FKDS-Kontrolle von Dialyseshunts sinnvoll ist, um Shuntthrombosen zu verhindern wird kontrovers diskutiert [16].

Einerseits wird bei der unbestritten hohen Treffsicherheit der Duplexsonographie in der Detektion von Shuntstenosen $[3,8]$ der Vorteil einer frühen Intervention bei der Diagnose eines drohenden Shuntverschlusses über Flussmessungen [2] sowie über Stenosedetektion und -graduierung [8] in sonographischen Verlaufsuntersuchungen hervorgehoben.

Dies bestätigt eine kürzlich publizierte Studie, die zeigt, dass ein SurveillanceProgramm zwar die Rate an Shuntinterventionen um 2,6\% steigert, dadurch die Rate an Shuntthrombosen aber auch um $8,4 \%$ reduziert wird [4]. Andererseits wird trotz der hohen Treffsicherheit $[3,10]$ in der Diagnose von Shuntproblemen (Aneurysma, Stenose, Teilthrombose) auch in einer großen Metaanalyse [15] sowie einer jüngst publizierten Zusammenstellung [9] gezeigt, dass dadurch das Risiko des Shuntfunktionsverlustes nicht reduziert wird, beziehungsweise das SurveillanceProgramme nicht gerechtfertigt sind.

Verlaufskontrollen von Kunststoffloops machen in Analogie zu Verlaufskontrollen von Kunststoffbypässen sicher keinen Sinn. Bei Dialyseloops gibt es dazu zwar keine Studienergebnisse, aber sowohl die Erfahrung als auch Studien über Kunststoffbypässe zeigen, dass routinemäBige Verlaufskontrollen die Verschlussrate nicht verbessert. Die in Surveillance-Programmen diagnostizierten Shuntstenosen 
können zu einer unterschiedlich aggressiven Reinterventionspolitik führen. Es stellt sich die Frage, inwieweit bei gut dialysierbarem Shunt Shuntstenosen dilatiert werden und inwieweit dann die Therapie sogar sekundär Probleme wie eine periphere Ischämie durch Steal-Phänomene verursachen kann. Es sollte jedoch frühzeitig bei Zeichen von Shuntproblemen (Shuntflussreduktion) sonographisch die Ursache gesucht werden und gezielt therapiert werden.

\section{Fazit}

- Wichtige Shuntprobleme (zu geringer Shuntfluss für Dialyse, periphere Ischämie, Armschwellung) können mithilfe der FKDS meist ohne weitere Untersuchungen einer adäquate Therapie zugeführt werden.

- Ein strukturiertes Vorgehen mit Spektralanalyse der shuntspeisenden Arterie sowie der Arterie distal der Anastomose mit und ohne manuelle Shuntkompression sowie der arteriovenösen Fistel kurz nach der Anastomose ermöglicht zeiteffizient die gezielte Diagnostik des shuntassoziierten Problems oder der Shuntstenose.

- Die Duplexsonographie ist valide in der Diagnostik von Shuntstenosen.

- Indirekte Stenosezeichen geben unter manueller Shuntkompression Hinweise auf eine vorgeschaltete Stenose in der shuntspeisenden Arterie.

- Ein pulsatiles Strömungssignal in der Arterie oder im proximalen Shuntabschnitt weist auf eine Shuntstenose im Verlauf oder eine zentralvenöse Abflussbehinderung hin.

\section{Korrespondenzadresse}

\section{Dr.W.Schäberle}

Klinik für Viszeral-, Gefäß-, Thoraxund Kinderchirurgie, Klinik am Eichert, Akademisches Lehrkrankenhaus der Universität Ulm Eichertstr. 3, 73035 Göppingen wilhelm.schaeberle@kae.de

\section{Einhaltung ethischer Richtlinien}

Interessenkonflikt. W. Schäberle und L. Leyerer geben an, dass kein Interessenkonflikt besteht.

Dieser Beitrag beinhaltet keine Studien an Menschen oder Tieren.

\section{Open Access}

This article is distributed under the terms of the Creative Commons Attribution License which permits any use, distribution, and reproduction in any medium, provided the original author(s) and the source are credited.

\section{Literatur}

1. Anaya-Ayala JE, Pettigrew CD, Ismail $N$ et al (2012) Management of dialysis access-associated "steal" syndrome with DRIL procedure: challenges and clinical outcomes. J Vasc Access 13:299-304

2. Bay WH, Henry ML, Lazarus JM et al (1998) Predicting hemodialysis access failure with color flow Doppler ultrasound. Am J Nephrol 18:296-304

3. Doelman C, Duijm LE, Liem YS et al (2005) Stenosis detection in failing hemodialysis access fistulas and grafts: comparison of color Doppler ultrasonography, contrast-enhanced magnetic resonance angiography and digital subtraction angiography. J Vasc Surg 42:739-746

4. Jiang S, Stewart G, Barnes E et al (2012) Effect of vascular access surveillance program on service provision and access thrombosis. Semin Dial (epub ahead of print)

5. Knox RC, Bergman SS, Hughes JD et al (2002) Distal revascularization - interval ligation: a durable and effective treatment for ischemic steal syndrome after hemodialysis access. J Vasc Surg 36(2):250-256

6. Kudlicka J, Kavan J, Tuka V et al (2012) More precise diagnosis of access stenosis: ultrasonography versus angiography. JVasc Access 13:310-314

7. Mickley V, Ranft S, Hollenbeck M, Haage P (2008) Leitlinie Shuntchirurgie. In: Deutsche Gesellschaft für Gefässchirurgie (Hrsg) Leitlinien zu Diagnostik und Therapie in der Gefässchirurgie. Springer, Heidelberg, S 167-178

8. Older RA, Gizienski TA, Wilkowski MJ et al (1998) Hemodialysis access stenosis: early detection with color Doppler US. Radiology 207:161-164

9. Paulson WD, Moist L, Lok CE (2013) Vascular access surveillance: case study of a false paradigm. Semin Dial (epub ahead of print)

10. Pietura R, Janczarek M, Zaluska W et al (2005) Colour Doppler ultrasound assessment of well-functioning mature arteriovenous fistulas for haemodialysis access. Eur J Radiol 55:113-119

11. Scali ST, Chang CK, Raghinaru D et al (2013) Prediction of graft patency and mortality after distal revascularization and interval ligation for hemodialysis access-related hand ischemia. J Vasc Surg 57:451-458

12. Schäberle W (2011) Ultrasonography in vascular diagnosis, 2. Aufl. Springer, Heidelberg, S 269-276

13. Schanzer $H$, Schwartz M, Harrington $E$, Haimov $M$ (1998) Treatment of ischemiadue to "steal " by arteriovenous fistula with distal artery ligation and revascularization. J Vasc Surg 7(6):770-773
14. Strauch BS, O'Conell RS, Geoly KL et al (1992) Forecasting thrombosis of vascular access with Doppler color flow imaging. Am J Kidney Dis 19:554557

15. Tonelli $\mathrm{M}$, James $\mathrm{M}$, Wiebe $\mathrm{N}$ et al (2008) Ultrasound monitoring to detect access stenosis in hemodialysis patients: a systematic review. Am J Kidney Dis 61:630-640

16. Vachharajani TJ (2012) Diagnosis of arteriovenous fistula dysfunction. Semin Dial 25:445-450

17. Zanow J, Petzold K, Petzold M et al (2006) Flow reduction in high-flow arteriovenous access using intraoperative flow monitoring. JVasc Surg 44(6):1273-1278 